\title{
Modelling of evaporation and dissolution of multicomponent oil droplet in shallow water
}

\author{
O. Samimi Abianeh ${ }^{1} \&$ C. P. Chen ${ }^{2}$ \\ ${ }^{1}$ Mechanical Engineering Department, \\ University of Alabama in Huntsville, USA \\ ${ }^{2}$ Chemical and Materials Engineering Department, \\ University of Alabama in Huntsville, USA
}

\begin{abstract}
A multicomponent evaporation and dissolution model was applied to study the dynamics of the mass transfer of dispersed oil in shallow waters. This model, in Lagrangian form, was coupled with the Princeton Ocean Circulation model (POM) for simulating oil droplet dispersion in an oceanic environment. The oil dispersion was modeled for a standard sea-mount shallow water environment. The most abundant petroleum hydrocarbons in the hydrocarbon-enriched of oil plum larger than $\mathrm{C}_{1}-\mathrm{C}_{5}$ were benzene, toluene, ethylbenzene, and total xylenes. Therefore, the oil surrogate, which consists of these components, is studied. The liquid-liquid equilibrium and vapor-liquid equilibrium equations are solved for evaluating the droplet life time. A rapid mixing model is used by reason of the shorter internal mass diffusion time scale in comparison to the droplet life time. Four different droplet sizes, $1.5,1,0.5$, and $0.1 \mathrm{~mm}$, are considered in this study. The droplet with a diameter of $1 \mathrm{~mm}$ has a shorter life time in this specific environment.

Keywords: multicomponent oil droplet dispersion, evaporation, dissolution, ocean circulation model, Rapid mixing model.
\end{abstract}

\section{Introduction}

Multicomponent liquid droplet size history during evaporation and dissolution influences the dynamic behavior of the droplets, whereas the variation of the composition determines the distribution of the fuel compounds within the environment, and also the droplet life time. The fundamental understanding of 
these processes is essential for modeling the evaporation and dissolution of fuel droplet.

Crude oil or fuels are typically composed of hundreds of complex compounds with different physical properties. Thus, the evaporation and dissolution of a multicomponent droplet is a complex process. There are several papers and reports dealing with droplet evaporation (e.g. [1-5]). Fuels are usually characterized by a single surrogate component for most of the evaporation models implemented in computational fluid dynamic codes since these models are computationally inexpensive. However, the single-component fuel models are not able to predict the complex behavior of complex fuels such as gasoline, diesel, and crude oil [2]. For predicting the real fuel distillation curve, usually more surrogate components are needed [3]. Multicomponent versus monocomponent models could increase the accuracy of predicting the evaporation/dissolution rate, fuel properties and also environmental emission by reason of different components. Typically, the oil spill was modeled by groups of major components (e.g. [7]), and each group of droplet consists of a monocomponent droplet. In this study each droplet is composed of multi components. One of the advantages of utilizing a multicomponent model instead of a monocomponent model is that the effect of components among each other in the mixture can be captured in the oil mixture.

Multicomponent fuel models are classified into two types: Discrete MultiComponent model (DMC) [3] and Continuous Multi Component model (CMC) [4]. The DMC approach treats a fuel with a limited number of components to represent the actual fuel distillation curve and thermal properties. The CMC model represents the fuel composition as a continuous distribution function of molecular weight or other appropriate parameters. The most abundant petroleum hydrocarbons in an oil spill (e.g. the Macondo well oil plum) larger than $\mathrm{C}_{1}-\mathrm{C}_{5}$ were benzene, toluene, ethylbenzene, and total xylenes at concentrations of up to $78 \mathrm{\mu gl}^{-1}[15,16]$. Therefore, the DMC model is utilized for predicting the life time of multicomponent droplet which consists of those major components.

Within the multicomponent droplet, previous studies can be classified into two general categories: the infinite conductivity model (Infinite Diffusion model or Rapid Mixing model) and the finite conductivity model [19]. The infinite conductivity model or Rapid Mixing model assumes a well-mixed droplet without a temperature gradient inside the droplet. Diffusion resistance within the droplet is zero for this approach. The model has reasonable results for slow evaporation processes, when droplet internal heat conduction and diffusion does not have a major effect on the internal temperature and concentration profiles [19]. This type of modeling is computationally efficient because there is no need to solve the mass and heat transfer for inside the droplet. In this study, the temperature of the oil droplet and surrounding environment (water or air) are assumed to be the same. Therefore, the heat transfer inside the droplet is not solved and also for mass transfer inside the droplet the Rapid Mixing model is utilized for the current oil spilling problem. The accuracy of this model is discussed in the Modeling formulation and Results sections. 
The ocean circulation model is solved by using the Princeton Ocean Model (POM) [8]. The major characteristics of the model are as follows: imbedded second moment turbulence closure sub-model, Arkawa $\mathrm{C}$ differencing scheme, the horizontal grid uses curvilinear orthogonal coordinates system; the horizontal time differencing is explicit whereas the vertical differencing is implicit. The droplet velocity and diameter are transferred between Lagrangian multicomponent mass transfer solver and POM for calculating the droplet Reynolds number, droplet position, and droplet life time.

\section{Modelling formulation}

The process of transport of molecular species of phase $\alpha$ into phase $\beta$ can be modeled by taking into account three resistances in series. The first transport step is the diffusion/convection of components within phase $\alpha$. The second transport step takes place across the interface. Finally, transport within the surrounding phase $\beta$ takes place by convection and diffusion. To simplify the analysis without losing generality, we assume that phase $\alpha$ is well mixed with no concentration gradients. The schematic of the droplet is shown in fig. 1.

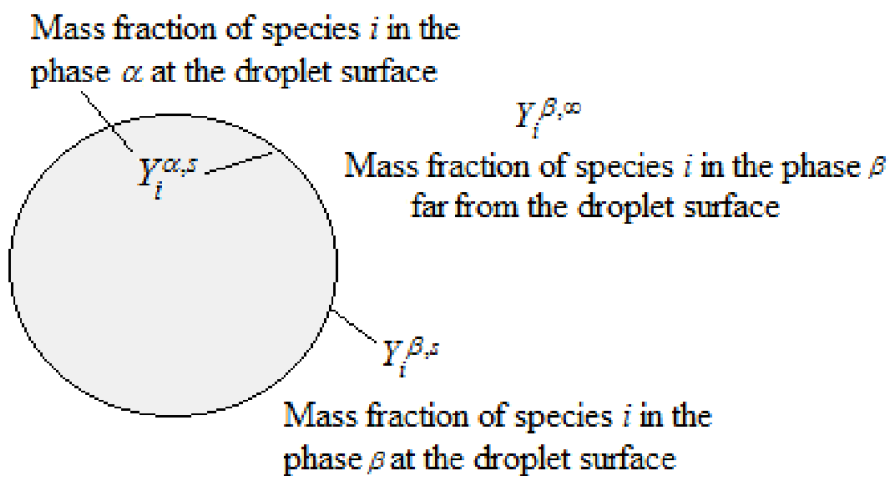

Figure 1: Schematic of evaporating/dissolution droplet.

The mass transfer equation for spherical symmetric droplet [10] can be written as

$$
\rho^{\alpha}\left(V^{\alpha, s}-\dot{r}\right) Y_{i}^{\alpha, s}-\rho^{\alpha} D_{i}^{\alpha} \nabla Y_{i}^{\alpha, s}=\rho^{\beta}\left(V^{\beta, s}-\dot{r}\right) Y_{i}^{\beta, s}-\rho^{\beta} D_{i}^{\beta} \nabla Y_{i}^{\beta, s},
$$

where $\rho, D, Y, V$, and $s$ are density, diffusivity, component mass fraction, velocity, and property at the droplet surface respectively. Eqn. (1) can be simplified by using the Rapid Mixing model and no accumulation condition on the surface of the droplet:

$$
\dot{m}_{i}=\dot{m} Y_{i}^{\alpha, s}=\dot{m} Y_{i}^{\beta, s}+J_{i}^{\beta, s}\left(Y_{i}^{\beta, s}-Y_{i}^{\beta, \infty}\right) .
$$


where: $J_{i}^{\beta, s}=S h_{i}^{\beta} \rho^{\beta} D_{i}^{\beta} / 2 r_{d}$, and $\dot{m}$ is the rate of mass transfer. Assuming phase $\alpha$ is insoluble in phase $\beta$, and at the distance far from the droplet surface $Y_{i}^{\beta, \infty}=0$, the total mass transfer rate could be derived as:

$$
\dot{m}=\frac{\sum_{i=1}^{N} J_{i}^{\beta, s} Y_{i}^{\beta, s}}{1-\sum_{i=1}^{N} Y_{i}^{\beta, s}},
$$

The vapor pressures are computed using Antoine's equation [18] and equilibrium molar fractions in the $\beta$-phase were computed using Raoult's Law [18]:

$$
x_{i}^{g, s}=x_{i}^{l, s} \frac{P_{i, v a p}(T)}{P_{a t m}}=K_{i}(T) x_{i}^{l, s},
$$

where $x_{i}^{g, s}$ and $x_{i}^{l, s}$ are the fuel component mole fraction in air (gas side) and droplet respectively. Mass transfer coefficients for hydrocarbons in air were computed using the expression for the Sherwood number developed by Torres et al. [9]. Diffusion coefficients and properties of air and hydrocarbons were estimated using [11] and [13]. For LLE (liquid-liquid equilibrium) calculation:

$$
x_{i}^{\beta, s}=K_{i}(T, P) x_{i}^{l, s},
$$

where $x_{i}^{\beta, s}$ and $x_{i}^{l, s}$ are the fuel component mole fraction in water and droplet respectively. Equilibrium distribution coefficient (or equilibrium distribution constant), $K_{i}(T, P)$, for hydrocarbons are determined from [12], and dispersion coefficients from the definition of the Sherwood number from [20].

The droplet velocity in a horizontal direction is assumed to be the same as the fluid velocity. However, the effect of buoyancy is considered for calculating the droplet vertical velocity:

$$
V_{p}(i, j, k)=V_{\text {fluid }}(i, j, k)+g(1-\beta) \tau,
$$

where $g$ is gravitational acceleration and:

$$
\beta=\frac{3}{2\left(\rho_{d} / \rho_{\text {fluid }}\right)+1}, \quad \tau=\frac{4 r_{d}^{2}}{3 \beta \vartheta},
$$

$\vartheta$ is the viscosity of fluid. Therefore, when the droplet is in water the slip velocity will be:

$$
V_{\text {slip }}(i, j, k)=g(1-\beta) \tau .
$$

When the droplet is at the surface of the water, the slip velocity is defined as the difference between air velocity (zero in this study) and the droplet velocity, thus:

$$
V_{\text {slip }}(i, j, k)=V_{\text {fluid }}(i, j, k),
$$

The slip velocity is used for calculating the Reynolds number.

Mass diffusion time scale is defined as the time required for a mass diffusion wave to penetrate from the droplet surface to its center. This time scale is in the order of:

$$
\tau_{M}=\frac{r_{d}^{2}}{D^{\alpha}},
$$

where $D^{\alpha}$ is mass diffusivity in liquid phase. The droplet life time could be estimated by: 


$$
\tau_{L}=O\left[\frac{\rho^{\alpha} r_{d}}{\dot{m}}\right]
$$

dimension of $\dot{m}$ is $\mathrm{kg} / \mathrm{m}^{2}$, from eqns. (3), and (11):

$$
\tau_{L}=O\left(\rho^{\alpha} r_{d} \times \frac{1-\sum_{i}^{n} Y_{i}^{\beta, s}}{\sum_{i}^{n} J_{i}^{\beta, s} Y_{i}^{\beta, s}}\right) .
$$

by assuming that the diffusivity of all of the components are the same. Moreover, having the same Sherwood number for all of the components, eqn. (12) could be more simplified by using eqn. (3):

$\tau_{L}=O\left(\rho^{\alpha} r_{d} \times \frac{1-\sum_{i}^{n} Y_{i}^{\beta, s}}{\sum_{i}^{n} J_{i}^{\beta, s} Y_{i}^{\beta, s}}\right)=O\left(\left(\frac{\rho^{\alpha}}{\rho^{\beta}}\right) \times\left(\frac{r_{d}^{2}}{\operatorname{sh}^{\beta} D^{\beta}}\right) \times\left(\frac{1-\sum_{i}^{n} Y_{i}^{\beta, s}}{\sum_{i}^{n} Y_{i}^{\beta, s}}\right)\right)$.

thus the ratio of internal mass diffusion time scale to the droplet life time (RTS: Ratio of Time Scales) could be calculated by using eqns. (10), and (13):

$$
R T S=\frac{\tau_{M}}{\tau_{L}}=O\left(\left(\frac{\rho^{\beta}}{\rho^{\alpha}}\right) \times\left(\frac{D^{\beta}}{D^{\alpha}}\right) \times\left(\frac{\sum_{i}^{n} Y_{i}^{\beta, S}}{1-\sum_{i}^{n} Y_{i}^{\beta, S}}\right) \times s h^{\beta}\right) .
$$

The ratios in eqn. (14) could be estimated for our problem by:

$$
R T S=\left(\frac{\tau_{M}}{\tau_{L}}\right) \cong\left\{\begin{array}{lr}
0.01, & \text { if } \beta \text { is water }(L L E) \\
0.1, & \text { if } \beta \text { is air }(V L E)
\end{array}\right\} .
$$

Therefore, we consider that the mass diffusion time from core of the droplet to the surface is short compared with lifetime. As a consequence, a nearly uniform species mass fraction is established quickly. Since the ratio of time scales (eqn. (15)) is a function of time, so the average of ratio of time scales over droplet life time could be defined by:

$$
R T S_{\text {average }}=\frac{1}{t} \int_{o}^{t} \frac{\tau_{M}}{\tau_{L}} d t=\frac{1}{N} \sum_{i=1}^{N}\left(\frac{\tau_{M}}{\tau_{L}}\right)_{i},
$$

where $t$ is droplet life time and $N$ is number of data over droplet life time. Eqns. (15), and (16) will be discussed in the results and discussions section.

\section{Boundary and initial conditions}

The model configuration is that of periodic $f$-plane channel; that is, two of the boundaries (at constant $y$ ) are solid, free slip walls, and the flow is assumed to be reentrant (periodic by time) in the channel stream-wise coordinate. The periodic boundary condition (forcing) is launched by periodically changing the boundary value for the bartropic stream function $(\psi)$ at one channel wall:

$$
\psi(x=0, t)=9.0 \sin \left(\frac{2 \pi t}{T}\right)
$$

where $T$ is 1 day and the stream function is zero at the other wall. The initial velocity is $1 \mathrm{~m} / \mathrm{s}$. A Gaussian seamount is placed at the center of the channel. The external dimensions of the channel are $345.511 \mathrm{~km}$ long $\times 259.265 \mathrm{~km}$ wide. The grid spacing varies smoothly from $4.0 \mathrm{~km}$ over the seamount to $8.0 \mathrm{~km}$ at the domain boundaries. The maximum depth in this problem is $50 \mathrm{~m}$. The model configuration is shown in fig. 2. The boundary condition and POM formulation 


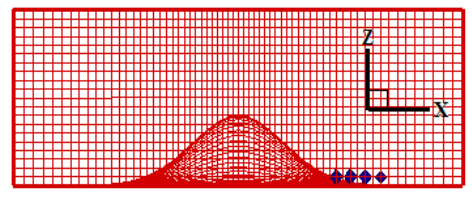

Figure 2: Model configuration.

of this problem were described with more details in $[14,17]$. The temperature of the air and water are assumed to be constant at $15^{\circ} \mathrm{C}$. the temperature of the droplet is the same as the water when it is inside of the water, and equal to air temperature when it is on the surface of the water.

The mixture composition of the multicomponent droplets is listed in Table 1, and they are derived from the experimental data of Camilli et al. [16]. Initial mixture density is $875 \mathrm{~kg} / \mathrm{m}^{3}$. Property of $\mathrm{m}$-xylene is utilized for the $\mathrm{m}, \mathrm{p}$-xylene family. For exploring the results, four droplet sizes ranging from $1.5 \mathrm{~mm}$ (Droplet \#1), $1.0 \mathrm{~mm}$ (Droplet \#2), $0.5 \mathrm{~mm}$ (Droplet \#3), to $0.1 \mathrm{~mm}$ (Droplet \#4) were investigated. The surrogated oil droplets were released at $50 \mathrm{~m}$ below the sea surface. Initially, the LLE calculation is activated, and when the droplet reaches the surface of the water vapor liquid equilibrium (VLE) calculation is activated.

Table 1: $\quad$ Mixture composition of droplet.

\begin{tabular}{|c|c|c|c|c|}
\hline Component & $\begin{array}{c}\text { Molecular } \\
\text { Weight }\end{array}$ & $\begin{array}{c}\text { Boiling } \\
\text { Temperature }\end{array}$ & $\begin{array}{c}\text { Mass Fraction } \\
{[16]}\end{array}$ & Formula \\
\hline benzene & 78.114 & 353.24 & 0.1369 & $\mathrm{C}_{6} \mathrm{H}_{6}$ \\
\hline toluene & 92.141 & 383.78 & 0.4109 & $\mathrm{C}_{7} \mathrm{H}_{8}$ \\
\hline ethylbenzene & 106.167 & 409.35 & 0.0684 & $\mathrm{C}_{8} \mathrm{H}_{10}$ \\
\hline m,p-xylene & 106.67 & $\begin{array}{c}\mathrm{m}: 412.27 \\
\mathrm{p}: 411.51\end{array}$ & 0.2739 & $\mathrm{C}_{8} \mathrm{H}_{10}$ \\
\hline o-xylene & 106.67 & 417.58 & 0.1099 & $\mathrm{C}_{8} \mathrm{H}_{10}$ \\
\hline
\end{tabular}

\section{Results and discussions}

The complexity of the coupling of the Lagrangian mass transferring solver and POM is that how often the data should be transferred between two solvers? The droplet life time is much smaller than time step of updating the data in POM. Capturing the history of more volatile components needs much smaller integration time-step. Generally the time step in POM (external time step) is about 1 day, and time step in Lagrangian mass transferring solver (internal time 
step) is less than one second $\left(\sim 10^{-3} \ldots 10^{-6} \mathrm{~s}\right)$. The other issue is that the velocity of the particle will affect the droplet life time due to changing Reynolds number. At the end of each external time step the velocity at droplet position will transfer to the Lagrangian solver. Subsequently by changing the external time step, the transferring velocity will be changed. Therefore, the computed droplet life time could be affected. The mass transfer due to the evaporation is larger than the dissolution. Therefore the time of reaching the surface should be computed precisely. For overcoming these issues, the time step of POM is changed to one second till the droplets exist in the domain.

The Lagrangian time steps for LLE calculation and VLE calculation are fixed to $10^{-4} \mathrm{~S}$ and $10^{-6} \mathrm{~S}$ respectively. Therefore, in each external time step, the Lagrangian solver will be run multiple times. At the surface of the water, the droplet mass transfer is governed by VLE calculation instead of LLE calculation. Therefore the rate of mass transfer increases as shown in droplet radius history in fig. 3. The time step of POM is changed to 5 second till the droplets exist in the domain.

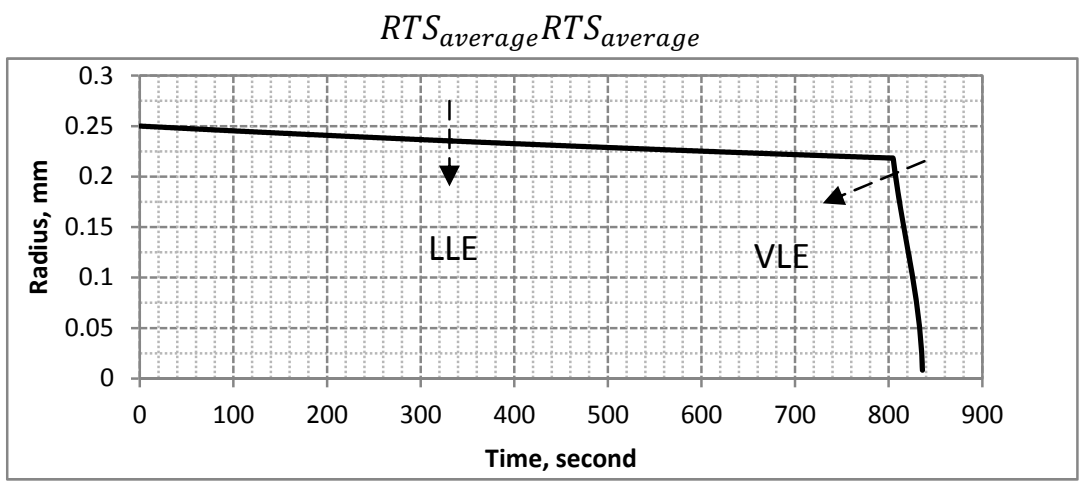

Figure 3: Droplet \#3 radius history.

The life time (evaporation and dissolution) of droplet with diameter of $1 \mathrm{~mm}$ is shorter than the other droplet sizes as shown in fig. 4. However, the life time of droplet\#1 and 2 are almost the same. Droplet \#4, with smallest diameter, has the largest life time among the others, since it takes longer time to reach the water surface compared to the other droplets. The Rate of mass transfer for dissolution is much smaller than evaporation. Therefore, the rate of droplet radius variation is larger during evaporation than dissolution as shown in figs. 3 , and 4.

The history of droplet composition for droplet \#1 is shown in fig. 5. The life time of more volatile components (e.g. benzene, and toluene) are shorter than the other components as expected. The mass fractions of components around the droplet (in water or air) are shown in fig. 6. The mass fractions of more volatile components (e.g. benzene, and toluene) are significant at start of evaporation and then they decrease as evaporation proceeds. The component mass fraction in the water is in the range of $10^{-4}$ to $10^{-6}$. 


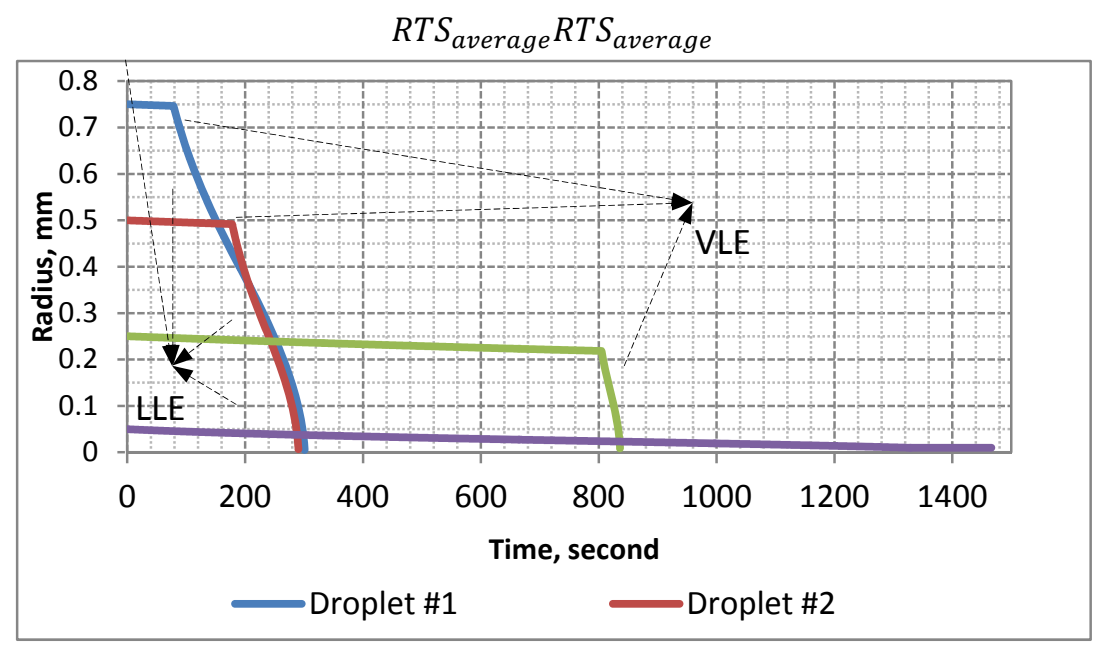

Figure 4: Droplet \#1, 2, 3, and 4 radius history.

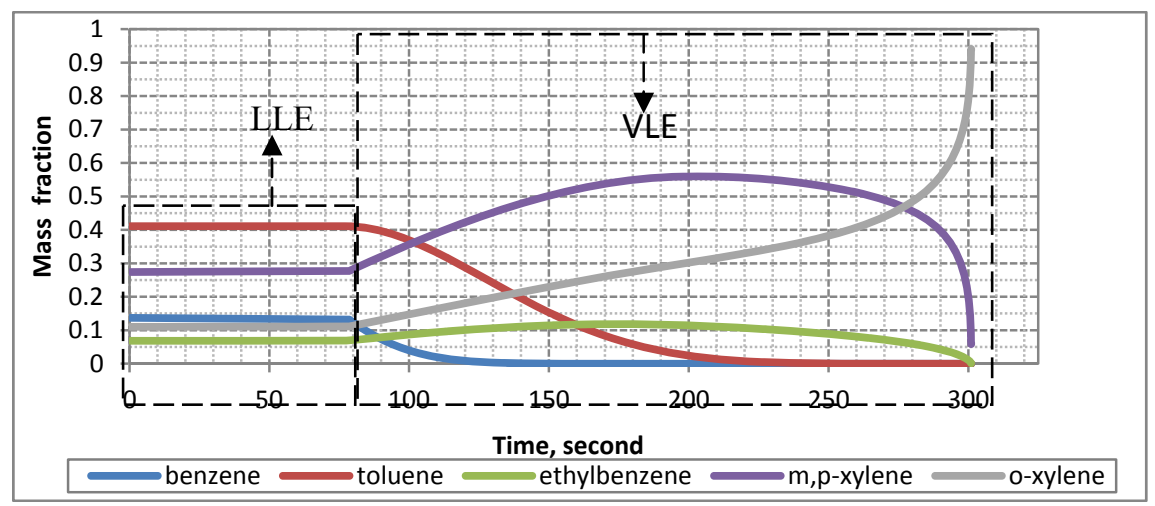

Figure 5: $\quad$ Composition mixture history for droplet $\# 1$.

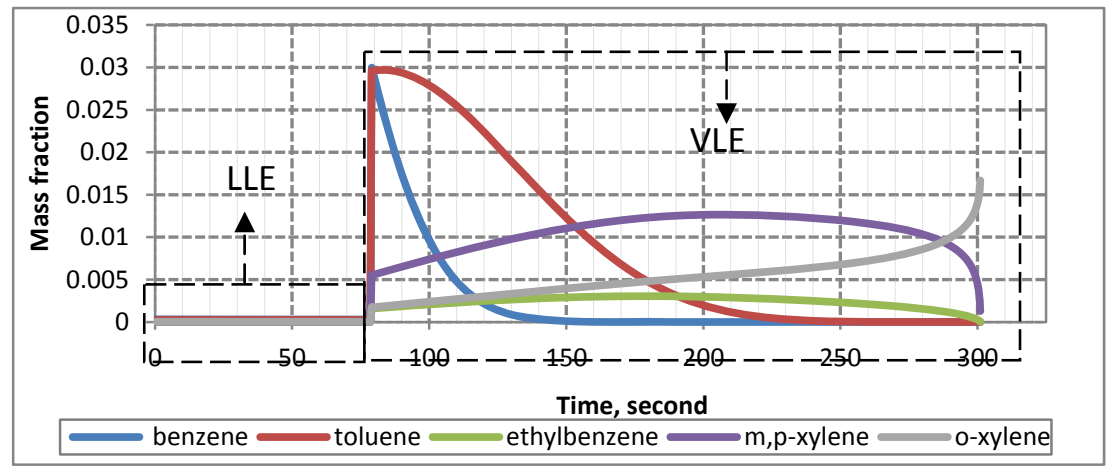

Figure 6: Composition mixture history around droplet \#1 (in water and air). 
As shown in Table 2 and fig. 7, the diffusion time scale could be $14 \%$ of droplet life time scale in air and 7\% when droplet is in water. Generally $R T S_{\text {average }}$ decreases when droplet diameter reduces. Due to decreasing rate of Sherwood number (Eqn. (14)). However, the life time of droplet\#3 on the surface of water is very short. As a result, $R T S_{\text {average }}$ increases in this case respect to droplet\#2 as shown in Table 2. Eqn. (10) overestimates the mass diffusion time scale inside of the droplet. In all cases internal circulation will decrease diffusion time scale [19] except for zero relative velocity between droplet and surrounding fluid (pure diffusion). Therefore, the ratio of time scales will be smaller than the numbers of Table 3. In conclusion, the simplification of Rapid Mixing model is valid for this problem.

Table 2: $\quad$ Average of ratio of two time scales (RTS $\left.S_{\text {average }}\right)$.

\begin{tabular}{|l|c|c|c|}
\hline & $\begin{array}{c}\text { RTS } \\
\text { LLerage }\end{array}$ & $\begin{array}{c}\text { RTS } \\
\text { average } \\
\text { VLE }\end{array}$ & $\begin{array}{c}\text { Diameter of the droplet when } \\
\text { it reaches water surface. }\end{array}$ \\
\hline Droplet\#1 & 0.068 & 0.098 & $1.490 \mathrm{~mm}$ \\
\hline Droplet\#2 & 0.045 & 0.084 & $0.984 \mathrm{~mm}$ \\
\hline Droplet\#3 & 0.018 & 0.14 & $0.433 \mathrm{~mm}$ \\
\hline Droplet\#4 & 0.0017 & \multicolumn{2}{|c|}{ It did not reach the water surface. } \\
\hline
\end{tabular}
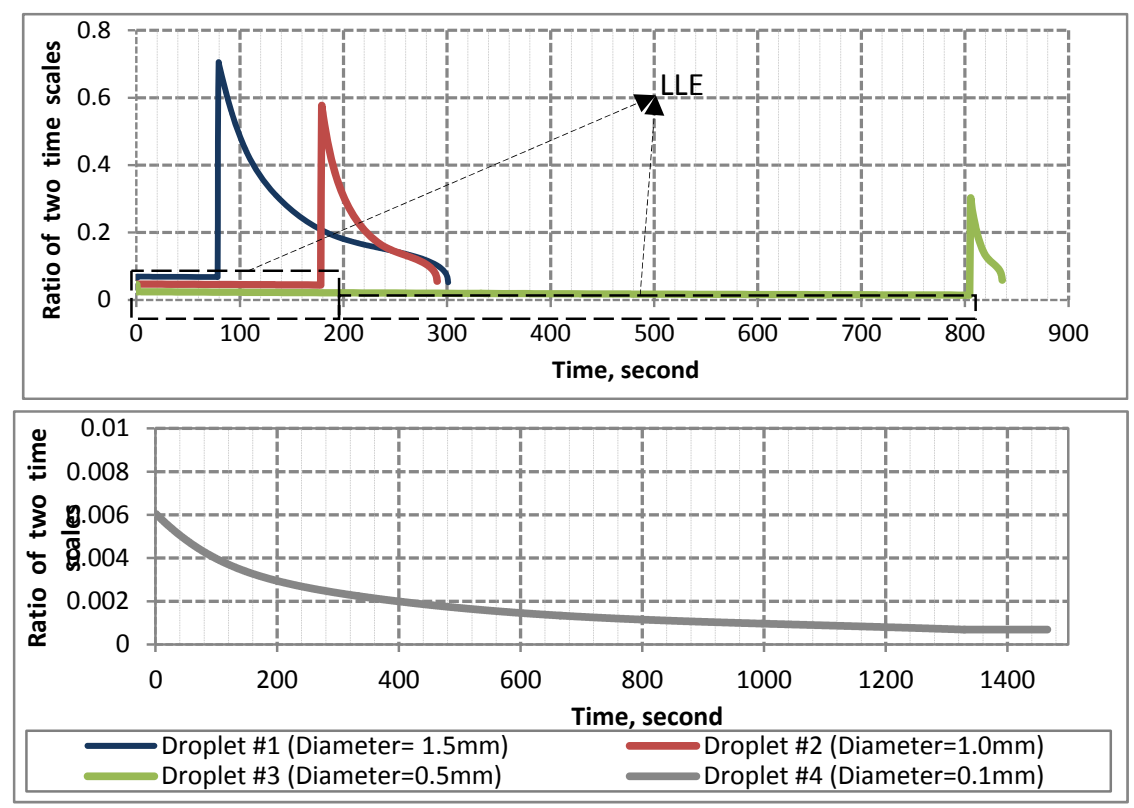

Figure 7: Ratio of two time scales for different droplet diameter.

The history of droplet positions is shown in fig. 8. As expected, the droplet with a larger size reaches the sea surface faster than the others due to having 
higher buoyancy. Eqn. (8) overpredicts the slip, and also rise-velocity of the big droplet. Chen and Yapa [21] predicted that the slip velocity (or rise velocity in their paper [21]) would not be bigger than $15 \mathrm{~cm} / \mathrm{s}$ for big droplet. Predicted slip velocities in this study are $60,27,6.7$, and $2.7 \mathrm{~cm} / \mathrm{s}$ for $1.5,1,0.5,0.1 \mathrm{~mm}$ respectively. Therefore eqn. (8) is not valid for the big droplet.

Table 3: $\quad R T S_{\text {average }} R T S_{\text {average }} R T S_{\text {average }}$ Timing of fig. 8.

\begin{tabular}{|c|c|c|c|c|c|c|c|}
\hline 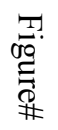 & 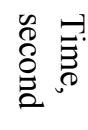 & 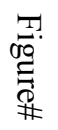 & 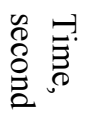 & 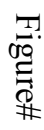 & 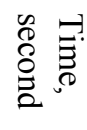 & 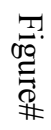 & 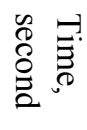 \\
\hline $\bar{a}$ & 0.0 & $\mathrm{~b}$ & 18.0 & $\mathrm{c}$ & 80.5 & d & 104 \\
\hline $\mathrm{e}$ & 288 & $f$ & 434 & $\mathrm{~g}$ & 799 & $\mathrm{~h}$ & 874 \\
\hline
\end{tabular}
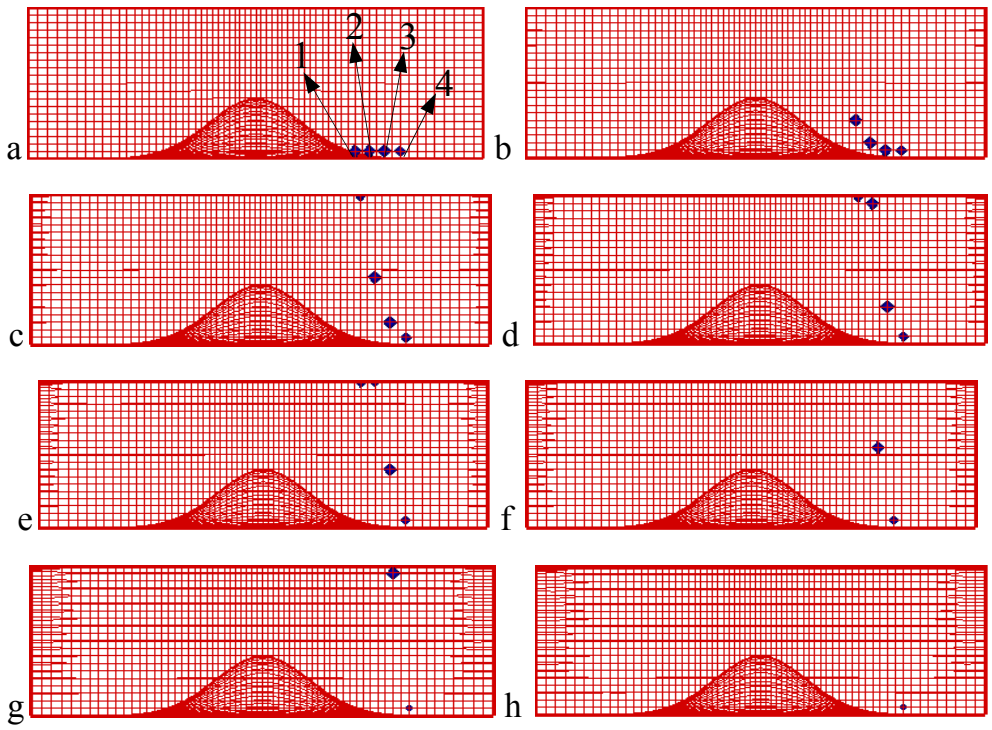

Figure 8: Position of droplets at different time. (See Table 3 for timing of each figure.)

\section{Conclusions}

A sigma-coordinate primitive equation ocean circulation model is employed to explore the problem of oil dispersion. A Lagrangian multicomponent mass transfer model is coupled with Princeton Ocean circulation Model (POM) for modeling oil droplet dispersion in oceanic environment. Summaries of the modeling results are:

- The evaporation and dissolution rate of more volatile component is greater than less volatile components. 
- The droplet with diameter of $1 \mathrm{~mm}$ has a shorter life time compared to other droplet sizes.

- Smaller droplets may not necessarily have shorter life time. As shown in this research, the smallest droplet with diameter of $0.1 \mathrm{~mm}$ has the longer life time.

- The Rapid Mixing Model is more applicable for smaller droplet.

- The mass diffusion time scale could be $14 \%$ of droplet life time when surrounding fluid is air. And this ratio is $7 \%$ when surrounding fluid is water.

- The buoyancy effect changes the droplet vertical velocity significantly. Therefore, droplet reaches the water surface faster.

\section{Acknowledgement}

This research was made possible by grant DISL T2-008-UAH from BP/The Gulf of Mexico Research Initiative.

\section{References}

[1] Balasubramanyam, M. S., Chen, C. P. and Trinh, H. P., A New Finite Conductivity Droplet Evaporation Model including Liquid Turbulence Effect. J. Heat Transfer, 129, pp. 1081-1086, 2007.

[2] Lippert, A. M., Modeling of Multi-Component Fuels with Application to Sprays and Simulation of Diesel Engine Cold Start. Ph.D. Thesis, University of Wisconsin-Madison, 1999.

[3] Ra, Y. and Reitz, R. D., A Vaporization Model for Discrete MultiComponent Fuel Sprays. In. J. Of Multiphase Flow, 35, pp. 101-117, 2009.

[4] Tamim, J., and Hallett, W. L. H., Continuous Thermodynamics Model for Multi-Component Vaporization. Chem. Eng. Sci. 50, pp. 2933-2942, 1995.

[5] Lippert, A. M. and Reitz, R. D., Modeling of Multi-Component Fuels using Continuous Distributions with Application to Droplet Evaporation and Sprays. SAE Paper 972882, 1997.

[6] Law, C. K. and Sirignano, W. A., Unsteady Droplet Combustion with Droplet Heating - II: Conduction Limit. Combustion and Flame, 28, pp. 175-186, 1977.

[7] Korotenk, K. A., Mamedov, R. M., Kontar, A. E. and Kortenco, L. A., Particle tracking method in the approach for prediction of oil slick transport in the sea: modelling oil pollution resulting from river input. Journal of Marine Systems, 48, pp. 159-170, 2004.

[8] Blumberg, A. F., and Mellor, G. L., A Description of a Three-dimensional Coastal Ocean Circulation Model, in Three-Dimensional Coastal Ocean Models. N. S. Heaps (Ed.), 1-16, American Geophysical Union, Washington, DC, 1987.

[9] Torres, D. J., O'Rourke, P. J. and Amsden, A. A., A Discrete Multicomponent Fuel Model. Journal Atomization and Sprays. 13, pp. 131-172, 2003. 
[10] Samimi Abianeh, O. and Chen, C. P., Advanced Model of Bi-Component Fuel Droplet Heating And Evaporating with Liquid Turbulence Effects at High Pressure. ASME, ASME 2011 Internal Combustion Engine Division Fall Technical Conference, ICEF2011-60040, pp. 109-119, 2011.

[11] Lide, D. R. and Kehiaian H. V., CRC Handbook of Thermophysical and Thermochemical Data. Boca Raton, FL: CRC Press, 1994.

[12] Maczynski, A, Wisniewska-Goclowska, B. and Goral, M., Recommended liquid-liquid equilibrium data. Part 1, Binary alkane-water systems. J. Phys. Chem. Ref. Data. 33, pp. 549-577, 2004.

[13] Cussler, EL., Diffusion, mass transfer in fluid systems. Cambridge, UK: Cambridge University Press, 1984.

[14] Mellor, G. L., L.-Y. Oey and T. Ezer, Sigma coordinate pressure gradient errors and the seamount problem. J. Atmos. Oceanic. Technol., 15(5), pp. 1122-1131, 1998.

[15] Christopher, M. Reddya, J. Samuel Areyb, Jeffrey S. Seewalda, Sean P. Sylvaa, Karin L. Lemkaua, Robert K. Nelsona, Catherine A. Carmichaela, Cameron P. McIntyrea, Judith Fenwickc, G. Todd Venturad, Benjamin A. S. Van Mooya and Richard Camillic, Composition and fate of gas and oil released to the water column during the Deepwater Horizon oil spill, Proc Natl Acad Sci USA, 10.1073/pnas.1101242108, 2011.

[16] Camilli, R., Reddy C. M., Yoerger, D. R., Van Mooy, B. A. S., Jakuba, M. V., Kinsey, J. C., McIntyre, C. P., Sylva, S. P. and Maloney, J. V., Tracking hydrocarbon plume transport and biodegradation at Deepwater Horizon. Science 330, pp. 201-204, 2010.

[17] Beckmann, A. and Haidvogel, D. B., Numerical simulation of flow around a tall isolated seamount. Part I: Problem formulation and model accuracy. J. Of physical oceanography, 23, pp. 1736-1753, 1993.

[18] Smith, J. M., Van Ness, H. C. and Abbott, M. M., Introduction to chemical engineering thermodynamic. Seventh ed., McGraw-Hill's, New York, 2005.

[19] Sirignano, W. A., Fluid Dynamics and Transport of Droplets and Sprays. Second ed., Cambridge University Press, New York, 2010.

[20] Pfannkuch, H.-O., Determination of the contaminant source strength from mass exchange processes at the petroleum-ground-water interface in shallow aquifer systems. Proceedings of the NWWA/API Conference on Petroleum Hydrocarbons and Organic Chemicals in Ground water Prevention, Detection and Restoration. National Water Well Association, Worthington, Ohio, pp. 111-129, 1984.

[21] Chen, F. and Yapa, P., A model for simulating deepwater oil and gas blowouts - Part II: Comparison of numerical simulations with "Deepspill" field experiments. Journal of Hydraulic Research.41 (4), pp. 353-365, 2002. 